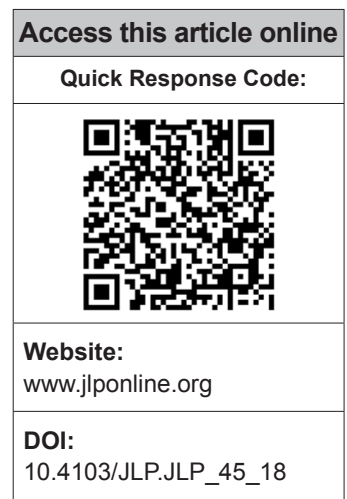

Department of Microbiology, Institute of Lab Medicine, Max Super Speciality Hospital, New Delhi, India

Address for correspondence: Dr. Bansidhar Tarai, Institute of Lab Medicine, Max Super Speciality Hospital, Saket, New Delhi - 110017 India.

E-mail: bansidhar.tarai@ maxhealthcare.com

Submission: 03-04-2018

Accepted: 05-08-2018

\title{
Evaluation of mean monocyte volume in septicemia caused by Salmonella species
}

\author{
Dilip Kumar, Madhusoodanan Sudha, Bansidhar Tarai, Poonam Das
}

\section{Abstract:}

OBJECTIVE: The objective was to study the significance of volume, conductivity, and scatter (VCS) parameters of monocytes in Salmonella infection.

MATERIALS AND METHODS: We selected peripheral blood samples of 52 patients whose blood culture was positive for Salmonella species (Salmonella typhi and Salmonella paratyphi A) and analyzed VCS parameters by subjecting them to differential count in hematology analyzer LH750 (Beckman Coulter). Out of these, we studied VCS parameters of monocytes in Salmonella infection and compared with two control groups (control 1: other infections and control 2: normal subjects).

RESULTS: Mean monocyte volume (MMV), standard deviation (SD) of MMV, and SD of mean channel monocyte conductivity were found to be significantly increased in Salmonella infection $(P<0.05)$ when compared with both controls. We propose a cutoff value of 185 for MMV with sensitivity and specificity of $80 \%$ and $73 \%$, respectively, to predict Salmonella infection.

CONCLUSIONS: MMV can be a useful tool for predicting Salmonella infection under appropriate clinical settings.

Key words:

Mean monocyte volume, monocytes, Salmonella, septicemia

\section{Introduction}

$V$ olume, conductivity, and scatter (VCS) parameters are used to obtain differential leukocyte count (DLC) in automated hematology analyzers (Beckman Coulter LH 750, India Pvt Ltd) based on Coulter's principle. VCS parameters include mean channel volume (MCV) and conductivity and scatter of neutrophils, lymphocytes, monocytes, and eosinophils. The corresponding standard deviation (SD) of these parameters is also obtained along with. These parameters can show variation according to various disorders affecting DLC.

Volume of white cells denotes size of the cell which is measured on the principle of impedance, i.e., using low voltage current

This is an open access journal, and articles are distributed under the terms of the Creative Commons Attribution-NonCommercial-ShareAlike 4.0 License, which allows others to remix, tweak, and build upon the work non-commercially, as long as appropriate credit is given and the new creations are licensed under the identical terms.

For reprints contact: reprints@medknow.com and measuring electric resistance changes which are produced by nonconducting cells in conducting medium. ${ }^{[1,2]}$ Conductivity is measured by radiofrequency produced by high-voltage electromagnetic current and that enters the cell and obtains information about the cell size and internal structure. Scatter is measured by making laser light pass through the hemodynamically focused stream of cells. Scatter is dependent on cell surface and internal structure like nuclear location and granules. ${ }^{[2]}$ The Coulter also provides the corresponding width of VCS parameters, namely SD of MCV, SD of mean channel conductivity, and SD of mean channel scatter. ${ }^{[3]}$

The usefulness of VCS parameters, especially mean neutrophil volume (MNV) and mean monocyte volume (MMV) for predicting acute bacterial infection, is already proven by previous studies. ${ }^{[4-6]}$ VCS parameters have

How to cite this article: Kumar D, Sudha M, Tarai B, Das P. Evaluation of mean monocyte volume in septicemia caused by Salmonella species. J Lab Physicians 2018;10:397-400. 
been studied in septicemic patients, but to the best of our knowledge, no significant study has been done on VCS parameters in case of Salmonella infection, particularly on MMV. Currently, the mainstay of diagnosis in enteric fever is blood culture and serological tests. ${ }^{[7,8]}$ When the blood investigations are sent to the laboratory, complete blood count (CBC) is almost always done as a part of workup. VCS parameters are automatically derived when the sample is subjected to $\mathrm{CBC}$ analysis, without additional cost. Considering the role of monocytes in the pathogenesis of Salmonella infections, ${ }^{[9-11]}$ we decided to study the change in the VCS parameters in Salmonella infections. We compared it with other infections and healthy controls to derive the sensitivity and specificity at particular cutoff value.

\section{Materials and Methods}

\section{Selection of cases}

This study was undertaken at a SuperSpeciality Hospital, New Delhi, India. In our prospective case-control study, we randomly selected 52 cases having blood culture positive for Salmonella species (including Salmonella typhi and Salmonella paratyphi $A$ ), irrespective of their age and sex. The peripheral blood samples from these cases were collected with proper aseptic precautions and analyzed for VCS parameters of monocytes. The patients' clinical records were also reviewed for correlation. All the samples for the study of VCS parameters were taken on the same day of blood culture sample collection.

As controls, we selected two groups for study of VCS parameters. One group included 82 septicemic cases positive for organisms other than Salmonella (Group 1), and the other group included 100 healthy individuals who came to the hospital for preventive health checkup and were found clinically normal (Group 2). The culture-positive cases in which the blood culture yielded bacteria likely to be contaminants were excluded from the study.

\section{Data collection}

For VCS parameters, $2 \mathrm{ml}$ of blood sample was collected in BD vacutainer (K2 ethylenediaminetetraacetic) under strict aseptic precautions by venepuncture method. Beckman Coulter LH750 was used for recording VCS parameters of white blood cells. For blood culture, two or more blood specimens were collected using sterile technique at separate sites, before antibiotic administration. BACT / ALERT $^{\circledast}$ disposable blood culture bottles were used for sample collection. The blood culture was performed in BACT / ALERT ${ }^{\circledR}$ microbial detection system.

\section{Statistical analysis}

All the analyses were performed using STATA software, version 9.0 (Stata Corp LLC College station, Texas, USA). Results were expressed as mean $\pm \mathrm{SD}$. Comparison between two means was performed by using Student's t-test and Mann-Whitney U-test. $P<0.05$ was considered statistically significant.

\section{Results}

\section{General data}

We collected the VCS parameters of 52 Salmonella cases and compared the same with VCS parameters of 82 cases positive for other organisms in culture (control Group 1) and with 100 healthy controls (control Group 2).

The average age of the Salmonella cases was 27.73, of the control Group 1 was 54.85, and control Group 2 was 42.34 . The male:female ratio of Salmonella cases was 1:1, of control Group 1 was 1.64:1, and of control Group 2 was 0.96:1.

The most common organisms cultured in the control Group 1 were Gram-negative bacilli, followed by Gram-positive cocci and yeasts. The most common bacteria cultured were Escherichia coli $(n=33)$, followed by Klebsiella pneumoniae $(n=20)$ [Table 1].

The average total leukocyte count (TLC) for Salmonella cases was $6.84 \times 10^{9} / \mathrm{L}$, while the same for control Group 1 , which included other infections, was $14.55 \times 10^{9} / \mathrm{L}$ and for healthy controls, it was $7.1 \times 10^{9} / \mathrm{L}$. The mean neutrophil percentage in Salmonella cases was 70.03\% and the same for control groups 1 and 2 was $81.95 \%$ and $60.7 \%$, respectively. The mean monocyte percentage in Salmonella, control Group 1, and control Group 2 was $7.17 \%, 3.79 \%$, and $6.49 \%$, respectively.

\section{Monocyte volume, conductivity, and scatter parameters}

The MMV and SD of MMV were found to be higher in Salmonella cases when compared to both the control groups [Table 2]. We statistically compared the MMV and SD of MMV of Salmonella cases with the control groups. We found a significant increase in MMV of

Table 1: Organisms cultured in control Group 1

\begin{tabular}{lc}
\hline Organisms & $n(\%)$ of cases \\
\hline Escherichia coli & $33(33)$ \\
Klebsiella pneumoniae & $20(20)$ \\
Pseudomonas aeruginosa & $5(5)$ \\
Acinetobacter baumannii & $4(4)$ \\
Enterococcus faecium & $4(4)$ \\
Staphylococcus aureus & $4(4)$ \\
Proteus mirabilis & $2(2)$ \\
Streptococcus pneumoniae & $2(2)$ \\
Candida parapsilosis & $2(2)$ \\
Enterococcus faecalis & $2(2)$ \\
Candida famata & $1(1)$ \\
Candida haemulonii & $1(1)$ \\
Enterobacter cloacae & $1(1)$ \\
Elizabethkingia meningosepticum & $1(1)$ \\
\hline
\end{tabular}


Salmonella cases when compared to other infections and healthy controls $(P<0.00)$. The SD of MMV and mean channel monocyte conductivity (MMC) also showed a significant increase when compared to both control groups $(P<0.05)$. Other monocyte parameters such as MMC, mean channel monocyte scatter (MMS), and SD of MMS were not significantly increased $(P>0.05)$ in Salmonella infection when compared to other infections. However, these parameters showed a significant increase when compared with healthy controls [Table 2].

\section{Sensitivity and specificity at the designated cutoff values}

Based on the above observations, we proposed a cutoff of 185 for MMV in Salmonella cases with a sensitivity of $80 \%$ and specificity of $73 \%$ when compared with control Group 1 and $98 \%$ when compared to control Group 2. The positive predictive value and negative predictive value for this cutoff are $66 \%$ and $85 \%$, respectively, when compared with other infections (Group 1). We also calculated the cutoff for SD of MMV. With a cutoff of 24, SD of MMV achieved a sensitivity of $80 \%$ and a specificity of $54 \%$ when compared with the control Group 1 and $96 \%$ when compared with Group 2 . The positive and negative predictive values were $53 \%$ and $81 \%$, respectively, when compared with control Group 1 . With a cutoff of 5, SD of MMC achieved a sensitivity of $77 \%$ and a specificity of $60 \%$ when compared with control Group 1 and $82 \%$ when compared with Group 2 and the positive and negative predictive values were $55 \%$ and $80 \%$, respectively, when compared with control Group 1 [Table 3].

\section{Discussion}

Typhoid fever, one of the major bacterial infections worldwide, is caused by human-adapted typhoidal serovars of Salmonella enterica, namely S. typhi, S. paratyphi $A$, S. paratyphi $B$, and S. paratyphi $C .{ }^{[7,12]}$ The highest incidence worldwide is found in Asia, particularly South-central and South eastern regions, and this is one of the most common organisms encountered in our country. ${ }^{[13]}$ The clinical diagnosis of typhoid fever is challenging due to diverse manifestations of the disease. At present, the diagnosis is based on clinical presentations, bacterial culture, serological markers, antigen detection, and molecular methods. The serological tests such as Widal can give rise to false-positive results and rapid tests such as Typhidot IgM also have a low sensitivity and specificity. ${ }^{[14]}$ The gold standard of diagnosis in typhoid fever is blood culture which is expensive, time-consuming (minimum $48 \mathrm{~h}$ ), and if the patient has taken any antibiotics before sample collection, may become false negative. ${ }^{[7]}$

Enteric fever clinically presents as high-grade fever, and $\mathrm{CBC}$ is one of the common tests done during the preliminary evaluation of fever. Findings such as leukopenia and neutropenia are seen in $15 \%-25 \%$ patients only. Leukocytosis is seen in children, in the first 10 days of disease, and in complications such as secondary infections. ${ }^{[12]}$ Hence, total leukocyte count and neutrophil percentage in these patients show high variation and of no use in supporting the diagnosis. ${ }^{[15]}$ In our study also, we

Table 2: Statistical comparison of volume, conductivity, and scatter parameters of monocytes in Salmonella with the control groups

\begin{tabular}{|c|c|c|c|c|c|}
\hline \multirow[t]{2}{*}{ Parameter } & \multicolumn{3}{|c|}{ Mean \pm SD } & \multicolumn{2}{|c|}{$\boldsymbol{P}$} \\
\hline & $\begin{array}{c}\text { Salmonella } \\
\text { cases }(n=52)\end{array}$ & $\begin{array}{l}\text { Control Group } 1 \\
\qquad(n=82)\end{array}$ & $\begin{array}{c}\text { Control Group } 2 \\
(n=100)\end{array}$ & $\begin{array}{c}\text { Salmonella versus } \\
\text { Group } 1\end{array}$ & $\begin{array}{c}\text { Salmonella versus } \\
\text { Group } 2\end{array}$ \\
\hline MMV & $194.94 \pm 9.84$ & $177.72 \pm 12.3$ & $169.95 \pm 5.11$ & 0.0000 & 0.0000 \\
\hline SD of MMV & $25.29 \pm 2.38$ & $24.06 \pm 4.68$ & $18.62 \pm 2.24$ & 0.0470 & 0.0000 \\
\hline MMC & $124.9 \pm 3.19$ & $124.5 \pm 5.2$ & $118.7 \pm 2.65$ & 0.5914 & 0.0000 \\
\hline SD of MMC & $5.89 \pm 1.11$ & $5.21 \pm 1.77$ & $4.27 \pm 0.40$ & 0.0137 & 0.0000 \\
\hline MMS & $83.57 \pm 4.49$ & $82.97 \pm 5.37$ & $87.08 \pm 3.41$ & 0.5087 & 0.0000 \\
\hline SD of MMS & $10.65 \pm 2.06$ & $11.46 \pm 3.307$ & $9.87 \pm 0.81$ & 0.1166 & 0.0010 \\
\hline
\end{tabular}

$\mathrm{MMV}=$ Mean channel monocyte volume, SD = Standard deviation, MMC = Mean channel monocyte conductivity, MMS = Mean channel monocyte scatter

Table 3: Sensitivity and specificity at the designated cutoff values of mean channel monocyte volume, standard deviation of mean channel monocyte volume, and standard deviation of mean channel monocyte conductivity for predicting Salmonella sepsis

\begin{tabular}{|c|c|c|c|c|c|c|}
\hline & Cutoff & Sensitivity (\%) & $\begin{array}{c}\text { Specificity when } \\
\text { compared to control } \\
\text { Group } 1(\%)\end{array}$ & $\begin{array}{l}\text { Specificity when } \\
\text { compared with } \\
\text { control Group } 2(\%)\end{array}$ & $\begin{array}{l}\text { Positive predictive } \\
\text { value when compared to } \\
\text { control Group } 1(\%)\end{array}$ & $\begin{array}{c}\text { Negative predictive } \\
\text { value when compared } \\
\text { to control Group } 1(\%)\end{array}$ \\
\hline \multirow[t]{3}{*}{ MMV } & 183 & 86 & 70 & 98 & 64 & 89 \\
\hline & 185 & 80 & 73 & 98 & 66 & 85 \\
\hline & 186 & 78 & 78 & 99 & 68 & 85 \\
\hline \multirow[t]{2}{*}{ SD of MMV } & 24 & 80 & 54 & 96 & 53 & 81 \\
\hline & 25 & 63 & 62 & 98 & 47 & 72 \\
\hline \multirow[t]{2}{*}{ SD of MMC } & 5 & 77 & 60 & 82 & 55 & 80 \\
\hline & 5.2 & 61 & 67 & 87 & 54 & 73 \\
\hline
\end{tabular}

$\mathrm{MMV}=$ Mean channel monocyte volume, SD = Standard deviation, MMC = Mean channel monocyte conductivity 
found an average TLC of $6.84 \times 10^{9} / \mathrm{L}$ for Salmonella cases with a range of $3 \times 10^{9} / \mathrm{L}-26 \times 10^{9} / \mathrm{L}$. The average neutrophil percentage was $70.03 \%$ with a range of $45 \%-90 \%$.

The VCS parameters are used to derive DLC in some automated hematology analyzers. These parameters assess the size of the cell and internal structure of the cells. The mononuclear phagocytic system plays an important role in the pathogenesis of Salmonella. In the peripheral blood also, bone marrow-derived monocytes contain these organisms. ${ }^{[9-11]}$ Hence, we studied the changes in the VCS parameters of monocyte in enteric fever to look for any specific changes. We found a significant change in MMV, SD of MMV, and SD of MMC, while MMC, MMS, and SD of MMS showed no significant change.

Here, in our study, we observed a significant increase in the MMV and SD of MMV in typhoid fever $(P<0.05)$ when compared with other infections and healthy controls.

MMV has been studied along with MNV in bacterial infections where Lee and Kim found a significant increase in MMV in sepsis and they proposed a cutoff of $\geq 175.5$ for MMV with sensitivity and specificity of $83.3 \%$ and $59.3 \%$, respectively. ${ }^{[5]}$ Mardi et al. also found a significant increase in MMV in their study on MNV and MMV in bacterial infections. ${ }^{[6]}$

We proposed a higher cutoff value of 185 for MMV to suspect Salmonella infection with a sensitivity of $80 \%$ and a specificity of $73 \%$ when compared with other infections (control Group 1). We calculated a cutoff of 24 for SD of MMV in our study with a sensitivity and a specificity of $80 \%$ and $54 \%$, respectively, when compared to Group 1. We also calculated a cutoff of 5 for SD of MMC with a sensitivity and a specificity of $77 \%$ and $60 \%$, respectively, when compared to Group 1. Since MMV, as compared to SD of MMV and MMC, carries a better specificity at a sensitivity of $80 \%$, we advocate the study of MMV parameter in Salmonella infection.

As compared to Typhidot IgM (rapid serological diagnosis) where the sensitivity and specificity had been reported as $75 \%$ and $60.7 \%$, respectively, ${ }^{[14]}$ MMV gives a better sensitivity and specificity as per our study without requirement of any additional serum sample. Can serological test be replaced by MMV parameter needs further study. We hypothesize that Salmonella infection causes complex intracellular changes resulting in increase in MMV and study of MMV can be a useful tool for diagnosing Salmonella infection under appropriate clinical settings.

\section{Conclusion}

Enteric fever is one of the most common causes of septicemia in developing countries due to poor sanitation conditions. Since the disease is associated with dreaded complications, it is essential to diagnose this at the earliest for better management and to prevent any complications. Though there are many tests available for diagnosing the typhoid fever, the role of MMV should also be taken into account. We propose a cutoff of 185 for MMV with a sensitivity of $80 \%$ and a specificity of $73 \%$ when compared with other infections to predict Salmonella septicemia, if there is clinical suspicion for the same. This test requires no additional workforce, instrumentation, or cost.

\section{Financial support and sponsorship} Nil.

\section{Conflict of interest}

There are no conflicts of interest.

\section{References}

1. Ward PC. The CBC at the turn of the millennium: An overview. Clin Chem 2000;46:1215-20.

2. Sullivan E. Hematology analyzer: From workhorse to thoroughbred. Lab Med 2006;37:274-8.

3. Lehner J, Greve B, Cassens U. Automation in haematology. Transfus Med Hemother 2006;34:328-39.

4. Chaves F, Tierno B, Xu D. Quantitative determination of neutrophil VCS parameters by the coulter automated hematology analyzer: New and reliable indicators for acute bacterial infection. Am J Clin Pathol 2005;124:440-4.

5. Lee AJ, Kim SG. Mean cell volumes of neutrophils and monocytes are promising markers of sepsis in elderly patients. Blood Res 2013;48:193-7.

6. Mardi D, Fwity B, Lobmann R, Ambrosch A. Mean cell volume of neutrophils and monocytes compared with C-reactive protein, interleukin- 6 and white blood cell count for prediction of sepsis and nonsystemic bacterial infections. Int J Lab Hematol 2010;32:410-8.

7. Wain J, Hosoglu S. The laboratory diagnosis of enteric fever. J Infect Dev Ctries 2008;2:421-5.

8. Olsen SJ, Pruckler J, Bibb W, Nguyen TM, Tran MT, Nguyen TM, et al. Evaluation of rapid diagnostic tests for typhoid fever. J Clin Microbiol 2004;42:1885-9.

9. Raffatellu M, Wilson RP, Winter SE, Bäumler AJ. Clinical pathogenesis of typhoid fever. J Infect Dev Ctries 2008;2:260-6.

10. Wijburg OL, Simmons CP, van Rooijen N, Strugnell RA. Dual role for macrophages in vivo in pathogenesis and control of murine Salmonella enterica var. Typhimurium infections. Eur J Immunol 2000;30:944-53.

11. Ohl ME, Miller SI. Salmonella: A model for bacterial pathogenesis. Annu Rev Med 2001;52:259-74.

12. Pegues DA, Miller SI. Salmonellosis. In: Longo DL, Kasper DL, Jameson JL, Fauci AS, Hauser SL, Loscalzo J, editors. Harrison's Principles of Internal Medicine. $18^{\text {th }}$ ed. Vol. 1. New York: McGraw-Hill Inc.; 2012. p. 1274-7.

13. Agarwal R, Gupta D, Ray P, Aggarwal AN, Jindal SK. Epidemiology, risk factors and outcome of nosocomial infections in a respiratory Intensive Care Unit in North India. J Infect 2006;53:98-105.

14. Keddy KH, Sooka A, Letsoalo ME, Hoyland G, Chaignat CL, Morrissey AB, et al. Sensitivity and specificity of typhoid fever rapid antibody tests for laboratory diagnosis at two sub-Saharan African sites. Bull World Health Organ 2011;89:640-7.

15. Khan M, Coovadia YM, Connoly C, Sturm AW. The early diagnosis of typhoid fever prior to the Widal test and bacteriological culture results. Acta Trop 1998;69:165-73. 